\title{
Flexible 19-Gauge Endobronchial Ultrasound-Guided Transbronchial Needle Aspiration Needle: First Experience
}

\author{
Chung Tyan ${ }^{\mathrm{a}}$ Priya Patel $^{\mathrm{a}} \quad$ Kasia Czarnecka ${ }^{\mathrm{a}} \quad$ Daniela Gompelmann ${ }^{\mathrm{c}}$ \\ Ralf Eberhardt ${ }^{c}$ Marc Fortin ${ }^{b}$ Paul MacEachern ${ }^{b}$ Christopher A. Hergott ${ }^{b}$ \\ Elaine Dumoulin ${ }^{b}$ Alain Tremblay ${ }^{b}$ Samuel V. Kemp ${ }^{d}$ Pallav L. Shah ${ }^{d}$ \\ Felix J.F. Herth ${ }^{c}$ Kazuhiro Yasufuku ${ }^{a}$

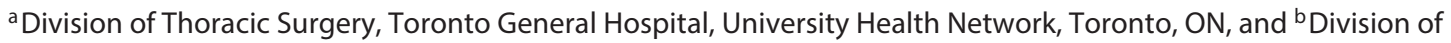 \\ Respiratory Medicine, University of Calgary, Calgary, AL, Canada; ' $\mathrm{C}$ epartment of Pneumology and Critical Care \\ Medicine, Translational Lung Research Center (TLRCH), Member of the German Lung Research Foundation (DZL), \\ Thoraxklinik University of Heidelberg, Heidelberg, Germany; ${ }^{\mathrm{d}}$ The National Institute for Health Research Unit, \\ Royal Brompton and Harefield NHS Foundation Trust, and Imperial College, London, UK
}

\section{Keywords}

EBUS-TBNA · Diagnostic bronchoscopy · Flexible 19-G EBUS needle $\cdot$ Mediastinal disease $\cdot$ Mediastinal lymph node . Hilar lymph node

\begin{abstract}
Background: Endobronchial ultrasound-guided transbronchial needle aspiration (EBUS-TBNA) is a well-established first-line invasive modality for mediastinal lymph node staging in lung cancer patients and in the diagnostic workup of patients with mediastinal adenopathy. With the current 21and 22-gauge (G) EBUS-TBNA needles, the procedure can be limited by the degree of flexibility in the needle and the size of the lumen in tissue acquisition. Objective: We report our initial experience with a first-generation flexible 19-G EBUSTBNA (Flex 19G; Olympus Respiratory America, Redmond, WA, USA) needle with regards to efficacy and safety. Methods: The Flex 19G EBUS-TBNA needle was used in 47 selected patients with enlarged hilar and/or mediastinal lymphade-
\end{abstract}

\section{KARGER}

(C) 2017 S. Karger AG, Basel

E-Mail karger@karger.com

www.karger.com/res nopathy at 3 centers. The standard Olympus EBUS scope with a 2.2-mm working channel was used in all cases. $R \boldsymbol{e}$ sults: The diagnostic yield of the Flex 19G needle according to clinical cytopathology reports was $89 \%(42 / 47)$. The diagnosis and their respective diagnostic yield with the Flex 19G EBUS-TBNA needle were malignancy $24 / 27$ (89\%), sarcoidosis $13 / 14$ (93\%), and reactive lymph node hyperplasia $5 / 6$ (83\%). The mean short axis of the sampled lymph nodes was $19 \pm 9 \mathrm{~mm}$. No complications occurred except for 1 instance of moderate bleeding, which did not require intervention beyond suctioning and subsequently resolved. All 13 patients diagnosed with adenocarcinoma by the 19-G needle had sufficient tissue for genetic testing. Conclusion: EBUSTBNA using the first-generation Flex $19 \mathrm{G}$ needle is feasible and safe with promising diagnostic yield while providing a greater degree of flexion with the Olympus EBUS scope. Additional clinical evaluations are warranted.

(c) 2017 S. Karger AG, Basel

Kazuhiro Yasufuku

Division of Thoracic Surgery, Toronto General Hospital, University Health Network 200 Elizabeth Street, 9N-957

Toronto, ON M5G 2C4 (Canada)

E-Mail kazuhiro.yasufuku@uhn.ca 


\section{Introduction}

Endobronchial ultrasound-guided transbronchial needle aspiration (EBUS-TBNA) is a well-established first-line invasive modality for mediastinal lymph node staging in lung cancer patients [1] and in the diagnostic workup of patients with mediastinal adenopathy [2]. The wide adaption of EBUS-TBNA has also shed some light on the limitation of the technology, namely the adequacy of fine-needle aspiration (FNA) samples in providing a definitive diagnosis for lymphoproliferative disease [3] and the shortfall in diagnostic accuracy of pulmonary sarcoidosis [4] in comparison to lung cancer diagnosis by EBUS-TBNA [5]. On the technical aspect, there is a limitation in the flexion at the distal tip of the EBUS bronchoscope due to the stiffness of the needle that lies within the channel, making access to mediastinal lymph nodes such as station $4 \mathrm{~L}$ and 10 more challenging [6].

Nineteen-gauge $(G)$ endoscopic ultrasound (EUS) needles have been utilized since 2005. In a review paper by Larghi and Fuccio [7], the performance of EUS biopsy by a $19-\mathrm{G}$ needle in 12 studies had a cumulative technical success and a diagnostic accuracy above $90 \%$. Yasuda et al. [8] demonstrated that $88 \%$ of the 152 patients diagnosed with lymphoma were able to be classified in accordance with the World Health Organization (WHO) system. Furthermore, a recent study that examines the role of a flexible 19-G EUS needle showcased the added benefit of a flexible needle in reaching target lesions that were otherwise difficult to reach by the same caliber of needle [9]. In the same study, in a cohort of 38 patients, the group was able to procure 100 and $95 \%$ satisfactory specimens for cytology and histology assessment, respectively. The benefits of a flexible 19-G EUS needle appear to address some of the technical limitations of EBUS-TBNA.

Recently, a flexible 19-G EBUS-TBNA needle (Flex 19G; Olympus Respiratory America, Redmond, WA, USA) was introduced. In our study, we aim to report the results of the first clinical experience of the Flex $19 \mathrm{G}$ needle in patients with a mediastinal and/or hilar lymphadenopathy/tumor.

\section{Materials and Methods}

\section{Patients}

The study is a retrospective analysis of all cases performed under a product evaluation. Three tertiary hospitals were involved in the Flex 19G needle evaluations: Toronto General Hospital, Foothills Medical Centre, and Heidelberg University Hospital. No upfront inclusion criteria were used, as cases were selected according to the clinical judgement of each bronchoscopists. In general, patients with enlarged mediastinal and/or hilar lymphadenopathy/ tumors were selected. The procedures were performed from September 2014 to March 2015. This study was conducted in accordance with the amended Declaration of Helsinki. The University Health Network Research Ethics Board number in conjunction with Heidelberg University Hospital is 16-5331. The Calgary Health Research Ethics Board number is 16-0184.

\section{EBUS-TBNA Procedure}

Conscious sedation was used during EBUS-TBNA with the exception of the Thoraxklinik (Heidelberg) where general anesthesia was used as the standard of care during EBUS-TBNA.

The EBUS-TBNA was performed with a flexible ultrasonic puncture bronchoscope with a linear scanning transducer with a frequency of 7.5 MHZ on the tip (convex probe-EBUS, BFUC180F; Olympus, Tokyo, Japan). It scans parallel to the insertion direction of the bronchoscope and is connected to a dedicated ultrasound scanner (EU-Y0005 \& EU-ME2; Olympus) with Doppler-flow imaging for the detection of blood vessels.

Once the target lesion was identified, the Flex 19G TBNA needle (Flex 19G; Olympus Respiratory America) was inserted into the $2.2-\mathrm{mm}$ working channel followed by the advancement of the sheath into the periphery of the endoscopic view. The internal stylet was kept in the needle during insertion. The needle was carefully placed just inferior to the proximal cartilage. Once the needle was advanced into the mass, the internal stylet was used to clear the tip of the needle. The 20-mL VacLoc (Merit Medical Systems, Inc., South Jordan, UT, USA) syringe was used to generate suction during the majority of passes. Nine different bronchoscopists were involved in the procedures.

\section{Sample Process}

Only selected cases performed in the Toronto General Hospital had rapid on-site evaluation prearranged. For all other cases, the samples collected from a TBNA needle were flushed into cytolyte fixative with an air-filled $20-\mathrm{mL}$ syringe [10]. With suspicion of infection or in the presence of necrotic material visually seen in the cytolyte fixative, subsequent needle sampling of the same lymph node would be flushed into a container filled with $10 \mathrm{~mL}$ of normal saline for microbiology culture and staining. Additional samples were sent for flow cytology assessment when lymphoma was suspected.

For cases chosen for rapid on-site evaluation, the stylet was used to push out the histologic cores inside of the needle for histologic evaluation. The aspirated material was smeared onto glass slides; air-dried smears were prepared and stained using the Romanowsky method. Once the cytopathologist deemed the specimen adequate, the appropriate ancillary studies were initiated.

Data Collected

Baseline patient and nodal characteristics were from chart review and review of CT imaging. Lymph nodes biopsied and procedural complications were recorded. The final diagnosis was based on EBUS-TBNA or further invasive investigation. All patient charts were reviewed to ensure the histologic diagnosis was consistent with the clinical course or any additional pathological sampling performed. In cases where the clinical course did not correspond to histologic diagnosis, the final diagnosis was changed to reflect the most likely diagnosis. 
Table 1. Baseline patient and lymph node characteristics and final diagnosis

\begin{tabular}{|c|c|}
\hline \multicolumn{2}{|l|}{ Patient characteristics } \\
\hline Total patients, $\%$ & 47 \\
\hline Mean age \pm standard deviation, years & $59 \pm 11$ \\
\hline Male, female, $n(\%)$ & $25,22(53,47)$ \\
\hline \multicolumn{2}{|l|}{ Lyhmph node/tumor characteristics } \\
\hline Lymph node station/tumor biopsied, $n(\%)$ & $125(100)$ \\
\hline $2 \mathrm{R}$ & $6(5)$ \\
\hline $2 \mathrm{~L}$ & $1(1)$ \\
\hline $4 \mathrm{R}$ & $34(27)$ \\
\hline $4 \mathrm{~L}$ & $16(13)$ \\
\hline 7 & $35(28)$ \\
\hline $10 \mathrm{R}$ & $12(10)$ \\
\hline $10 \mathrm{~L}$ & $3(2)$ \\
\hline $11 \mathrm{R}$ & $10(8)$ \\
\hline $11 \mathrm{~L}$ & $7(6)$ \\
\hline Tumor, \% & $1(1)$ \\
\hline Short axis (range), $\mathrm{mm}$ & $19(5-80)$ \\
\hline
\end{tabular}

\section{Statistical Analysis}

The study sample includes all cases performed at the 3 centers participating in the evaluations and as such was determined by the supply and availability of the devices from the manufacturer. Descriptive statistics are used to present the data. Continuous variables such as patient age or lesion size were summarized as means and standard deviations. Categorical variables such as sex, lymph node station, diagnosis, and complications were expressed as frequencies and proportions. The datasets were compiled with $\mathrm{Mi}$ crosoft Excel.

\section{Results}

A total of 47 patients with undifferentiated mediastinal or hilar lymphadenopathy have undergone EBUS-TBNA with the Flex $19 \mathrm{G}$ needle (Table 1). This includes 13 patients from Toronto General Hospital, 14 patients from Foothill Medical Centre, and 20 patients from Heidelberg University Hospital. Nine experienced bronchoscopists took part in the EBUS-TBNA procedures with the Flex $19 \mathrm{G}$ needle in their respective centers. One hundred and twenty-five target lesions (mean 2.7/patient) were sampled with an average short axis of $19 \mathrm{~mm} \pm 9$ (Table 2). Based on the clinical requirement, thoracic lymph node station $2 \mathrm{R}, 2 \mathrm{~L}, 4 \mathrm{R}, 4 \mathrm{~L}, 7,10 \mathrm{R}, 10 \mathrm{~L}, 11 \mathrm{R}$, and $11 \mathrm{~L}$ were amenable to biopsy (Table 1 ). In 16 patients at Toronto General Hospital and Foothill Medical Centre, a 22-G TBNA needle (NA-201SX-4022; Olympus) was used for technical comparison; 3 out of 3 station $4 \mathrm{~L}$ were success-
Table 2. Diagnostic yield, complication rate, and molecular testing viability

\begin{tabular}{ll}
\hline Diagnosis & $\begin{array}{l}\text { Diagnostic by Flex 19G } \\
\text { needle, } n / N(\%)\end{array}$ \\
\hline Malignant & $24 / 27(89)$ \\
$\quad$ Pulmonary adenocarcinoma & $14 / 14(100)$ \\
Pulmonary squamous cell carcinoma & $3 / 3(100)$ \\
Small-cell lung cancer & $3 / 3(75)$ \\
Lymphoma & $4 / 4(100)$ \\
Others (mesothelioma and nuclear & \\
protein in testis midline carcinoma) & $0 / 2(0)$ \\
Benign & $18 / 20(90)$ \\
Sarcoidosis & $13 / 14(93)$ \\
Reactive lymphocytosis & $5 / 7(83)$ \\
Complication & $1 / 47(2)$ \\
Molecular testing for adenocarcinoma & $14 / 14(100)$ \\
\hline
\end{tabular}

fully punctured by both needles, and 2 out of 2 station 10L were successfully punctured by the Flex 19G needle only. However, with increased flexibility, the Flex 19G needle was more prone to bending if the needle was pushed out of the sheath and encountered cartilage on its trajectory. One complication was reported as bleeding after sampling a station $11 \mathrm{R}$ lymph node; no further intervention was required beyond suctioning.

Overall, biopsy with the Flex 19G needle achieved a diagnosis in 42 patients ( $89 \%$, Table 2). Five patients were nondiagnostic. Final diagnosis was achieved by mediastinoscopy in 2 patients, brushing biopsy during the same procedure in 1 patient, sampling with a $22-\mathrm{G}$ needle during the same procedure in 1 patient, and clinical diagnosis made by further imaging in 1 patient.

Malignancy was the final diagnosis in 27 patients (57\%). Three patients with the final diagnosis of nuclear protein in testis midline carcinoma, mesothelioma, and small-cell lung cancer were not diagnostic. All 14 patients with a final diagnosis of adenocarcinoma were diagnostic with the Flex 19G needle. Molecular testing for epidermal growth factor receptor (EGFR) could be performed in all samples with a final diagnosis of adenocarcinoma in Canadian centers $(n=6)$. Heidelberg University Hospital's analysis of adenocarcinoma included EGFR, KRAS, anaplastic lymphoma kinase (ALK), BRAF, RET, and ROS1, and was possible in all 8 samples. Three out of 4 lymphoma diagnoses were subtyped: 1 patient's cytology demonstrated Hodgkin lymphoma and subsequently required mediastinoscopy for subtyping.

Benign diagnoses were made in 20 patients. The diagnostic yield was $93 \%$ for sarcoidosis (13/14). All patients 
Fig. 1. Endobronchial ultrasound-guided transbronchial needle aspiration scope max angulation with the Flex 19-G needle (left) and the 21-G needle (right).
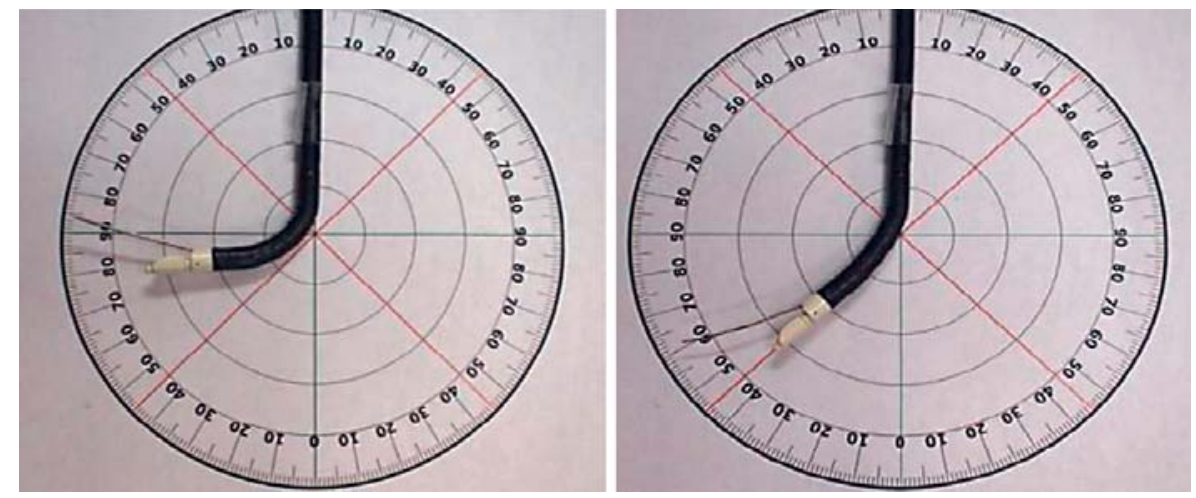

with reactive lymphocytosis were followed up radiologically to ensure accuracy of the diagnosis. In the single patient not diagnosed with the Flex 19G needle, the diagnosis was made with a $22-\mathrm{G}$ needle during the same procedure.

\section{Discussion}

The overall diagnostic yield (89\%) from biopsy with the Flex 19G needle is comparable to EBUS-TBNA studies performed with $21-\mathrm{G}$ and $22-\mathrm{G}$ needles $[11,12]$. While the sample size of this study is not powered to make generalizations regarding the diagnostic yield of lymphoma, it does show excellent promise for further studies in the area of lymphoproliferative disease diagnosis and subtyping by EBUS-TBNA. EUS-FNA study using the 19-G needle obtained a diagnosis of lymphoproliferative disease in $97 \%$ (147/152) and subtyped $89 \%$ of the samples obtained in accordance with the WHO system [8]. In the study by Yasuda et al. [8] with an EUS 19-G needle, enough samples were normally obtained in 2 passes for pathologically and cytological examinations. A study size of this magnitude with high diagnostic yield and subtyping has not been replicated in EBUS-TBNA studies with smaller bore needles $[13,14]$. The implication of a 19-G EUSFNA needle in the diagnosis of lymphoproliferative disease offers an exciting area of future research using subsequent product iterations.

Molecular testing for EGFR and ALK in advanced non-small cell lung cancer is now endorsed by the American Society of Clinical Oncology [15] and European Society of Medical Oncology [16]. As first-line therapies, EGFR tyrosine kinase inhibitor chemotherapy and ALK inhibition significantly increased progression-free survival compared to conventional chemotherapy in treating metastatic tumor with respective activating mutation [17-20]. The clinical importance of molecular testing is well established. The debate regarding the adequacy of cytological versus histologic samples for molecular testing is ongoing [16], but to date, studies have demonstrated excellent results for the detection of EGFR and ALK mutations with EBUS-TBNA [21,22]. The result of our data echoes the findings in previous studies with $100 \%$ of the samples from adenocarcinoma satisfactory for EGFR and ALK testing. The 19-G EUS needle was able to obtain tissue sufficient for histologic analysis as well [9]. With the advent of more targeted chemotherapy, it would be interesting to investigate if the Flex 19G needle can reliably procure tissue satisfactory for histologic examination and if this confers an advantage to meet the demand of increasing molecular testing.

A systematic review looking at the diagnostic yield of EBUS-TBNA in diagnosing sarcoidosis by Agarwal et al. [4] showed an overall diagnostic yield of $79 \%$. Similar yield was echoed in a recent randomized controlled study comparing endoscopic-guided biopsy versus conventional bronchoscopy biopsy in diagnosing sarcoidosis [23]. Our study demonstrated a diagnostic yield of $93 \%$ (13/14). A possible explanation for the higher diagnostic yield lies with the larger lumen of the Flex 19G needle, increasing the likelihood of larger tissue samples and the chance of identifying noncaseating granulomas. Although more pathology studies are needed to confirm the correlation between larger needles and tissue sample, our study suggests a signal for improving the diagnostic yield of sarcoidosis with the Flex 19G needle.

Historically, the thoracic lymph nodes accessible to EBUS-TBNA include stations 2-4, 7, and 10-12 [24]. In our study, station 10L was only accessible by the Flex 19G needle in 2 cases, while with the 22-G needle, the ultrasound probe was unable to come in contact with the re- 
spiratory mucosal wall to generate images for guidance. In both situations, the degree of flexion at the tip of the bronchoscope was increased with the Flex $19 \mathrm{G}$ within the working channel (Fig. 1). The clear implication of a more flexible needle is greater access to target lesions such as those in the upper lobe station 12. However, the greater flexibility and larger gauge did come with increased technical difficulties. As alluded to in the Results section, the Flex $19 \mathrm{G}$ needle was more prone to bending once the needle came into contact with cartilage on its way out of the sheath. In comparison to the stiffer and smaller $22-G$ or 21-G needles, it was harder to slide in between cartilage to reach target lesions. As more experience was gained using the Flex 19G needle, the operators paid greater attention to the anticipated trajectory of the needle. Nevertheless, the first version of the Flex 19G gave greater flexibility at the price of lesser penetration.

In our first experience with the Flex 19G EBUS-TBNA needle in 47 patients, we reported 1 minor complication (2\%). In relative comparison, large-scale registries with data from Japan [25] and the United States [26] reported an EBUS-TBNA complication rate of just above 1 percent. The clear difference between our study and the registries is the significant difference in the sample number, and therefore it is difficult to draw a conclusion. While no previous experience has compared the $19-\mathrm{G}$ to the $22-\mathrm{G}$ needle in a bronchoscopic procedure, a EUS-FNA study with 117 patients randomized to either $19 \mathrm{G}$ or $22 \mathrm{G}$ for the biopsy of pancreatic lesion showed a lower complication rate in the $19 \mathrm{G}$ group [27]. From an operator's perspective, sampling with a larger bore needle does affect one's selection of the needle entry site in order to minimize the perceived potential for risk. However, it is debatable if the extra attention paid to the placement of the needle is the reason for the low complication rate versus simply a manifestation of learning bias.

The limitations of the study are the sample size and lack of consistent comparison with conventional needles. However, this is a proof of concept study with strong emphasis on looking at the safety and general yield, which we believe the study has adequately addressed.

In summary, EBUS-TBNA using the first-generation Flex 19G needle is feasible and safe with a promising diagnostic yield while providing a greater degree of flexion with the Olympus EBUS scope. The potential for a larger sample to be procured by a minimally invasive procedure in the thorax offers potential for research in areas of subtyping lymphoproliferative disease and further improving the yield of EBUS for sarcoidosis and molecular testing of NSCLC. Furthermore, with its greater level of flexibility, the application of the Flex $19 \mathrm{G}$ needle may address biopsy in anatomical areas that are historically technically difficult.

\section{Acknowledgements}

We like to thank Olympus Respiratory America for providing the Flex 19G needles and Xavier Gonzalez for providing initial technical support. We thank all research assistants of the different institutes for helping with the submission of ethic board approval. We are grateful for all clinical information provided by the patients and referring physicians.

\section{References}

1 Yasufuku K, Pierre A, Darling G, de Perrot M, Waddell T, Johnston M, et al: A prospective controlled trial of endobronchial ultrasoundguided transbronchial needle aspiration compared with mediastinoscopy for mediastinal lymph node staging of lung cancer. J Thorac Cardiovasc Surg 2011;142:1393-1400.e1.

2 Navani N, Lawrence DR, Kolvekar S, Hayward M, McAsey D, Kocjan G, et al: Endobronchial ultrasound-guided transbronchial needle aspiration prevents mediastinoscopies in the diagnosis of isolated mediastinal lymphadenopathy: a prospective trial. Am J Res Crit Care Med 2012;186:255-260.

3 Rao AK, Ben-Or S, Bowling MR: Bronchoscopic myths and legends transbronchial needle aspiration of mediastinal and hilar lymph nodes in the diagnosis of lymphoma. Clin Pulm Med 2014;21:50-52.
4 Agarwal R, Srinivasan A, Aggarwal AN, Gupta D: Efficacy and safety of convex probe EBUS-TBNA in sarcoidosis: a systematic review and meta-analysis. Respir Med 2012; 106:883-892.

5 Gu P, Zhao Y-Z, Jiang L-Y, Zhang W, Xin Y, Han B-H: Endobronchial ultrasound-guided transbronchial needle aspiration for staging of lung cancer: a systematic review and metaanalysis. Eur J Cancer 2009;45:1389-1396.

6 Annema JT: Size matters. J Thorac Cardiovasc Surg 2015;149:942.

7 Larghi A, Fuccio L: Endoscopic ultrasoundguided fine needle aspiration: how to obtain a core biopsy? Endosc Ultrasound 2014;3:7111.
8 Yasuda I, Goto N, Tsurumi H, Nakashima M, Doi S, Iwashita T, et al: Endoscopic ultrasound-guided fine needle aspiration biopsy for diagnosis of lymphoproliferative disorders: feasibility of immunohistological, flow cytometric, and cytogenetic assessments. Am J Gastroenterol 2011;107:397-404.

9 Varadarajulu S, Bang JY, Hebert-Magee S: Assessment of the technical performance of the flexible 19-gauge EUS-FNA needle. Gastrointest Endosc 2012;76:336-343.

10 van der Heijden EH, Casal RF, Trisolini R, Steinfort DP, Hwangbo B, Nakajima T, et al: Guideline for the acquisition and preparation of conventional and endobronchial ultrasound-guided transbronchial needle aspiration specimens for the diagnosis and molecular testing of patients with known or suspected lung cancer. Respiration 2014;88:500-517. 
11 Silvestri GA, Gonzalez AV, Jantz MA, Margolis ML, Gould MK, Tanoue LT, et al: Methods for staging non-small cell lung cancer: diagnosis and management of lung cancer, 3rd ed: American College of Chest Physicians evidence-based clinical practice guidelines. Chest 2013;143:e211S-e250S.

12 Navani N, Lawrence DR, Kolvekar S, Hayward M, McAsey D, Kocjan G, et al: Endobronchial ultrasound-guided transbronchial needle aspiration prevents mediastinoscopies in the diagnosis of isolated mediastinal lymphadenopathy. Am J Respir Crit Care Med 2012;186:255-260.

13 Moonim MT, Breen R, Fields PA, Santis G: Diagnosis and Subtyping of de novo and relapsed mediastinal lymphomas by endobronchial ultrasound needle aspiration. Am J Respir Crit Care Medicine 2013;188:12161223.

14 Grosu HB, Iliesiu M, Caraway NP, Medeiros LJ, Lei X, Jimenez CA, et al: Endobronchial ultrasound-guided transbronchial needle aspiration for the diagnosis and subtyping of lymphoma. Ann Am Thorac Soc 2015;12: 1336-1344.

15 Leighl NB, Rekhtman N, Biermann WA, Huang J, Mino-Kenudson M, Ramalingam SS, et al: Molecular testing for selection of patients with lung cancer for EGFR and anaplastic lymphoma kinase tyrosine kinase inhibitors: American Society of Clinical Oncology Endorsement of the College of American Pathologists/International Association for the study of lung cancer/association for molecular pathology guideline. J Clin Oncol 2014;32: 3673-3679.
16 Kerr KM, Bubendorf L, Edelman MJ, Marchetti A, Mok T, Novello S, et al: Second ESMO consensus conference on lung cancer: pathology and molecular biomarkers for nonsmall-cell lung cancer. Ann Oncol 2014;25: 1681-1690.

17 Rosell R, Carcereny E, Gervais R, Vergnenegre A, Massuti B, Felip E, et al: Erlotinib versus standard chemotherapy as first-line treatment for European patients with advanced EGFR mutation-positive non-small-cell lung cancer (EURTAC): a multicentre, open-label, randomised phase 3 trial. Lancet Oncol 2012; 13:239-246.

18 Zhou C, Wu Y-L, Chen G, Feng J, Liu X-Q, Wang C, et al: Erlotinib versus chemotherapy as first-line treatment for patients with advanced EGFR mutation-positive non-smallcell lung cancer (OPTIMAL, CTONG-0802): a multicentre, open-label, randomised, phase 3 study. Lancet Oncol 2011;12:735-742.

19 Mitsudomi T, Morita S, Yatabe Y, Negoro S, Okamoto I, Tsurutani J, et al: Gefitinib versus cisplatin plus docetaxel in patients with nonsmall-cell lung cancer harbouring mutations of the epidermal growth factor receptor (WJTOG3405): an open label, randomised phase 3 trial. Lancet Oncol 2010;11:121-128.

20 Solomon BJ, Mok T, Kim D-W, Wu Y-L, Nakagawa K, Mekhail T, et al: First-line crizotinib versus chemotherapy in ALK-positive lung cancer. N Engl J Med 2014;371:21672177.

21 Folch E, Yamaguchi N, VanderLaan PA, Kocher ON, Boucher DH, Goldstein MA, et al: Adequacy of lymph node transbronchial needle aspirates using convex probe endobronchial ultrasound for multiple tumor genotyping techniques in non-small-cell lung cancer. J Thorac Oncol 2013;8:1438-1444.
22 Casadio C, Guarize J, Donghi S, Di Tonno C, Fumagalli C, Vacirca D, et al: Molecular testing for targeted therapy in advanced nonsmall cell lung cancer: suitability of endobronchial ultrasound transbronchial needle aspiration. Am J Clin Pathol 2015;144:629634.

23 von Bartheld MB, Dekkers OM, Szlubowski A: Endosonography vs conventional bronchoscopy for the diagnosis of sarcoidosis: the GRANULOMA randomized clinical trial. JAMA 2013;309:2457-2464.

24 Medford ARL, Bennett JA, Free CM, Agrawal $S$ : Endobronchial ultrasound-guided transbronchial needle aspiration (EBUS-TBNA): applications in chest disease. Respirology 2010;15:71-79.

25 Asano F, Aoe M, Ohsaki Y, Okada Y, Sasada $\mathrm{S}$, Sato S, et al: Complications associated with endobronchial ultrasound-guided transbronchial needle aspiration: a nationwide survey by the Japan Society for Respiratory Endoscopy. Respir Res 2013;14:50.

26 Eapen GA, Shah AM, Lei X, Jimenez CA, Morice RC, Yarmus L, et al: Complications, consequences, and practice patterns of endobronchial ultrasound-guided transbronchial needle aspiration. Chest 2013;143:10441053.

27 Song TJ, Kim JH, Lee SS, Eum JB, Moon SH, Do Hyun Park, et al: The prospective randomized, controlled trial of endoscopic ultrasound-guided fine-needle aspiration using $22 \mathrm{G}$ and $19 \mathrm{G}$ aspiration needles for solid pancreatic or peripancreatic masses. Am J Gastroenterol 2010;105:1739-1745. 